\title{
ORIGINAL ARTICLE Extreme polyandry aids the establishment of invasive populations of a social insect
}

\author{
G Ding ${ }^{1,2}, \mathrm{H} \mathrm{Xu}^{1}, \mathrm{BP}$ Oldroyd $^{3}$ and RS Gloag ${ }^{3}$
}

Although monandry is believed to have facilitated the evolution of eusociality, many highly eusocial insects have since evolved extreme polyandry. The transition to extreme polyandry was likely driven by the benefits of within-colony genetic variance to task specialization and/or disease resistance, but the extent to which it confers secondary benefits, once evolved, is unclear. Here we investigate the consequences of extreme polyandry on the invasive potential of the Asian honey bee, Apis cerana. In honey bees and other Hymenoptera, small newly founded invasive populations must overcome the genetic constraint of their sex determination system that requires heterozygosity at a sex-determining locus to produce viable females. We find $A$. cerana queens in an invasive population mate with an average of 27 males (range 16-42) that would result in the founding queen/s carrying $75 \%$ of their source population's sex alleles in stored sperm. This mating frequency is similar to native-range Chinese A. cerana (mean 29 males, range 19-46). Simulations reveal that extreme polyandry reduces the risk, relative to monandry or moderate polyandry, that colonies produce a high incidence of inviable brood in populations that have experienced a founder event, that is, when sex allele diversity is low and/or allele frequencies are unequal. Thus, extreme polyandry aids the invasiveness of $A$. cerana in two ways: (1) by increasing the sex locus allelic richness carried to new populations with each founder, thereby increasing sex locus heterozygosity; and (2) by reducing the population variance in colony fitness following a founder event.

Heredity (2017) 119, 381-387; doi:10.1038/hdy.2017.49; published online 23 August 2017

\section{INTRODUCTION}

Monandry generates high relatedness among siblings, increasing the indirect fitness benefits to offspring of helping their mothers to reproduce. As such, monandry is posited to be a critical precondition for the evolution of reproductive altruism, such as the worker sterility seen in the social insects (Hughes et al., 2008; Boomsma, 2009; Cornwallis et al., 2010). Yet, polyandry has evolved independently in at least nine extant clades of social insects with advanced sociality (Hughes et al., 2008), including some ants (Fjerdingstad and Boomsma, 2000; Kronauer et al., 2007; Barth et al., 2014), wasps (Goodisman et al., 2002; Hanna et al., 2014) and bees (Palmer and Oldroyd, 2000; Tarpy et al., 2004, 2015). Indeed, the average mate number of queens exceeds 10 in some species, earning the label 'extreme polyandry' (Palmer and Oldroyd, 2000; Tarpy and Page, 2001; Barth et al., 2014). This shift from ancestral monandry to multiple mating was likely driven by one or more factors that favour polyandry, but only occurs in species where workers have lost their reproductive totipotency (ie, they have lost the ability to mate or produce eggs, Hughes et al., 2008). Postulated benefits of polyandry include a reduction in variance in brood viability among colonies (Page, 1980; Page and Marks, 1982; Palmer and Oldroyd, 2000), a decrease in intracolonial kin conflict over reproduction (Ratnieks, 1988; Mattila et al., 2012), insurance against inbreeding and the effects of reduced effective population sizes caused by colonial life (Barth et al., 2014) and an increase in the genetic diversity of workers, in turn improving a colony's disease resistance (Baer and Schmid-Hempel, 1999; Seeley and Tarpy, 2007) and the division of labour among its workers (Jones et al., 2004; Mattila and Seeley, 2007; Oldroyd and Fewell, 2008).

Polyandry may also confer secondary benefits to those lineages in which it has evolved. Secondary benefits are unlikely to have been major drivers favouring the transition to polyandry or even extreme polyandry, but nevertheless have significant consequences for a species' ecology or behaviour. Here we consider one such benefit of polyandry, and of extreme polyandry in particular: an increase in a species' potential for ecological invasiveness. Invasiveness is facilitated by an ability to found new populations from small numbers of individuals (Berggren, 2001). Yet, many Hymenopteran insects (ants, bees, wasps) have an inbuilt genetic handicap that hampers their ability to establish new populations from few founders: their sex determination mechanism (van Wilgenburg et al., 2006). Sex determination in Hymenoptera is governed by heterozygosity at one, or few, genes (Beye et al., 2003; Hasselmann and Beye, 2004; Heimpel and de Boer, 2008). Diploid individuals heterozygous at the 'sex locus', or loci, develop into females, whereas haploid individuals develop as males. Diploid individuals with identical sex alleles develop as diploid males, but these do not survive to adulthood or cannot sire normal offspring (Cook and Crozier, 1995; Beye et al., 2003). Because of this system of sex determination, populations need a large number of sex

${ }^{1}$ College of Plant Protection, China Agricultural University, Beijing, China; ${ }^{2}$ Key Laboratory of Pollinating Insect Biology of the Ministry of Agriculture, Institute of Apicultural Research, Chinese Academy of Agricultural Sciences, Beijing, China and ${ }^{3}$ Behaviour and Genetics of Social Insects Laboratory, School of Life and Environmental Sciences, The University of Sydney, Sydney, New South Wales, Australia

Correspondence: Dr RS Gloag, Behaviour and Genetics of Social Insects Laboratory, School of Life and Environmental Sciences, The University of Sydney, Sydney, New South Wales 2006, Australia.

E-mail: ros.gloag@sydney.edu.au

Received 27 April 2017; revised 27 June 2017; accepted 12 July 2017; published online 23 August 2017 
alleles at equal frequency to ensure that the risk of sex locus homozygosity is low (Cook and Crozier, 1995). Insufficient allelic diversity in small populations of Hymenopteran insects can lead to their extinction (Zayed and Packer, 2005). Polyandry might therefore aid invasive potential in two ways. First, a polyandrous queen that establishes a new population has a greater chance of bringing with her the diversity of sex alleles required to keep the incidence of diploid males low and for the nascent population to avoid extinction. Second, following a founder event, polyandry might reduce the population's variance in diploid male production (DMP) among colonies. Assuming that colonies incur a fitness cost from high DMP, this reduction in variance will in turn increase the proportion of colonies in the population that survive to reproduce. Polyandry's effect on the variance of brood viability has been examined for large populations with sex alleles at equilibrium (Page, 1980; Page and Marks, 1982; Palmer and Oldroyd, 2000), but its relative benefit is less intuitive in populations where allele number and frequency have been distorted by founder effects.

Here we investigate the consequences of extreme polyandry for the invasive potential of the Asian honey bee, Apis cerana. The average queen mating frequency for this species in its native range has been previously estimated at 18 (Oldroyd et al., 1998). In the past half century, A. cerana has proven highly invasive, spreading outside its native range of Asia and India into Papua New Guinea, the Torres Strait Islands, the Solomon Islands and, most recently, mainland Australia, where it arrived in 2007 (Koetz, 2013; Figure 1). Genetic evidence from the Australian population is consistent with it being derived from just one (or very few) founding colonies (Gloag et al., 2016). We first estimate the mating frequency of $A$. cerana queens in Australia's invasive population, and use the observed mating frequencies to calculate the average proportion of a source population's sex alleles that would be expected to be carried to a new location by each founding queen. We also compare invasive range mating frequencies with those of a nativerange population in China to assess whether invasive queens exhibit especially high polyandry. Second, we use simulations to estimate the effect of extreme polyandry by queens on the variance in brood viability (ie, diploid male production) experienced by colonies following a founder event, namely when sex allele richness is low and allele frequency skew is high (Gloag et al., 2016).

\section{MATERIALS AND METHODS}

\section{Worker genotyping}

We removed mature worker pupae from A. cerana colonies with naturally mated queens collected in 2014-2015: 9 hived colonies from Guangdong, China (native range), and 10 wild colonies from Cairns, Australia (invasive range) (Figure 1; $n=84-96$ pupae per colony). We extracted DNA from one hind leg per pupae using a 5\% Chelex solution protocol (Walsh et al., 1991). We amplified DNA in $5 \mu \mathrm{l}$ reactions $\left(1 \times\right.$ reaction buffer, $2.5 \mathrm{~mm} \mathrm{MgCl}_{2}$, $0.16 \mathrm{~mm}$ dNTP mixture, $0.32-0.8 \mu \mathrm{M}$ of fluorescent dye-labelled primers, Sigma-Aldrich, St Louis, MO, USA, 0.4 units Taq polymerase and $1 \mu \mathrm{l}$ extracted DNA) and resolved PCR products using an ABI3130xl genetic analyzer (Applied Biosystems, Foster City, CA, USA). We genotyped native-range colonies at 5-6 microsatellite loci known to be highly polymorphic in their population (A077, Ac146, Ap050, Ap056, Ap055 and Ac194, Solignac et al., 2003; 7-15 alleles per locus). Because genetic diversity in the invasive population is very low (Gloag et al., 2016), we genotyped these colonies at 12 loci to compensate for the relatively low polymorphism per locus (Ac1, Ac3, Ac26, Ac27, Ac32, Ac35, Ac30, A14, A107 and B124, Solignac et al., 2003; Takahashi et al., 2009; 2-4 alleles per locus; Ap43 and the complementary sex determiner, csd, 6-7 alleles per locus, Gloag et al., 2016). All loci genotyped in this study and the corresponding number of alleles detected per population are given in Supplementary Table 1.

\section{Calculation and analysis of mating frequency}

We used COLONY v2.0.6.1 (Jones and Wang, 2010) to assign worker paternity and estimate the observed mating frequency per queen $(k$, the number of subfamilies within each colony) using default parameters (genotyping errors $=$ $0-0.1 \%$, full-likelihood analysis and different random number seed). We first ran the program inputting both worker genotypes and the deduced queen genotypes for each colony. After visual inspection of the output of father genotypes, we combined any fathers with the same genotype and ran analyses again, inputting the inferred father genotypes into the data set. The output of this analysis provided a minimum number of full-sib families and thus the observed mating frequency of each queen. Because males may contribute unequally to offspring paternity, we also calculated effective mating frequency (m), following Nielsen et al. (2003):

$$
m=\frac{(n-1)^{2}}{\sum_{i=1}^{k} p_{i}^{2}(n+1)(n-2)+3-n}
$$

where $n$ is the number of sampled offspring of a queen, $k$ is the observed mating frequency and $p$ is the proportional contribution to the brood of the $i$ th male. Effective mating frequency will equal absolute mating frequency if all males father an equal proportion of brood and the sample size is high.

The nondetection errors for each population, $d_{p}$ (that is, the probability of failing to distinguish the offspring from two different fathers because they shared the same genotype by chance), were estimated based on population allele frequencies by multiplying the summed squared allele frequencies for each locus (Boomsma and Ratnieks, 1996) using the formula:

$$
d p=\prod_{1}^{j} \sum q_{i}^{2}
$$

where $q_{i}$ denotes the allele frequency of the $i$ th allele at the $j$ th locus.

We estimated the mean and range number of sex alleles that would be expected to be carried to a new location by a single founding A. cerana queen. For this estimation, we assumed a randomly mating source population containing $20 \mathrm{sex}$ alleles at equal frequency (previous studies have estimated native-range populations to contain 17-22 alleles, Hasselmann et al., 2008 ; Gloag et al., 2016). We then ran 1000 sample iterations, where each iteration we sampled $k$ alleles with replacement and determined the number of unique alleles present in the sample, with $k$ equal to the average observed mating frequency from our Australian samples.

Finally, we determined whether observed queen mating frequencies differed between native Chinese and invasive Australian populations using the $t$-test. Observed mating frequencies did not deviate significantly from normality (test for normality, Shapiro-Wilk test: $P=0.597$ ).

Simulations of variance in brood viability in invasive populations To determine how extreme polyandry influences the variance in DMP under the conditions typical of an invasive population, we performed Monte Carlo simulations by sampling from a multinomial distribution with parameters $k$ (observed mating frequency of queens) and $s$ (number of sex alleles). We estimated the mean and s.d. of DMP for all $k \leqslant 30$, based on 1000 iterations for each value of $k$, for each of three scenarios: (1) sex allele number is high $(s=20)$ and allele frequencies are equal (1/20), as in native-range populations; (2) sex allele number is low $(s=7)$ and alleles are at skewed frequencies, as occurs in invasive populations immediately following a founder event (Gloag et al., 2016); and (3) sex allele number is low $(s=7)$ but allele frequencies are equal, as occurs in invasive populations that experienced a past founder event but are now established with sex allele frequency at equilibrium (Gloag et al., 2016). Allele numbers and frequencies for our simulated invasive populations were based on observed values from the Australian invasion by A. cerana. Sex alleles were highly skewed in 2008, the first year following introduction $(s=7$, sex alleles of frequencies: $A_{1}=0.31, A_{2}=0.17, A_{3}=0.01, A_{4}=0.23, A_{5}=0.26$, $\left.A_{6}=0.01, A_{7}=0.01\right)$, but rapidly approached equilibrium frequency $(1 / 7)$ in 2015, at 8 years post invasion (Gloag et al., 2016).

To visualize how DMP variance influences the absolute DMP under each scenario, we chose three illustrative levels of polyandry: $k=1$ (monandry), $k=6$ (moderate polyandry) and $k=27$ (extreme polyandry), and estimated the proportion of colonies per population containing $\geqslant 25$ and $\geqslant 35 \%$ diploid male brood. We consider these DMP frequencies (equivalent to 1 in 4 , or 1 in 


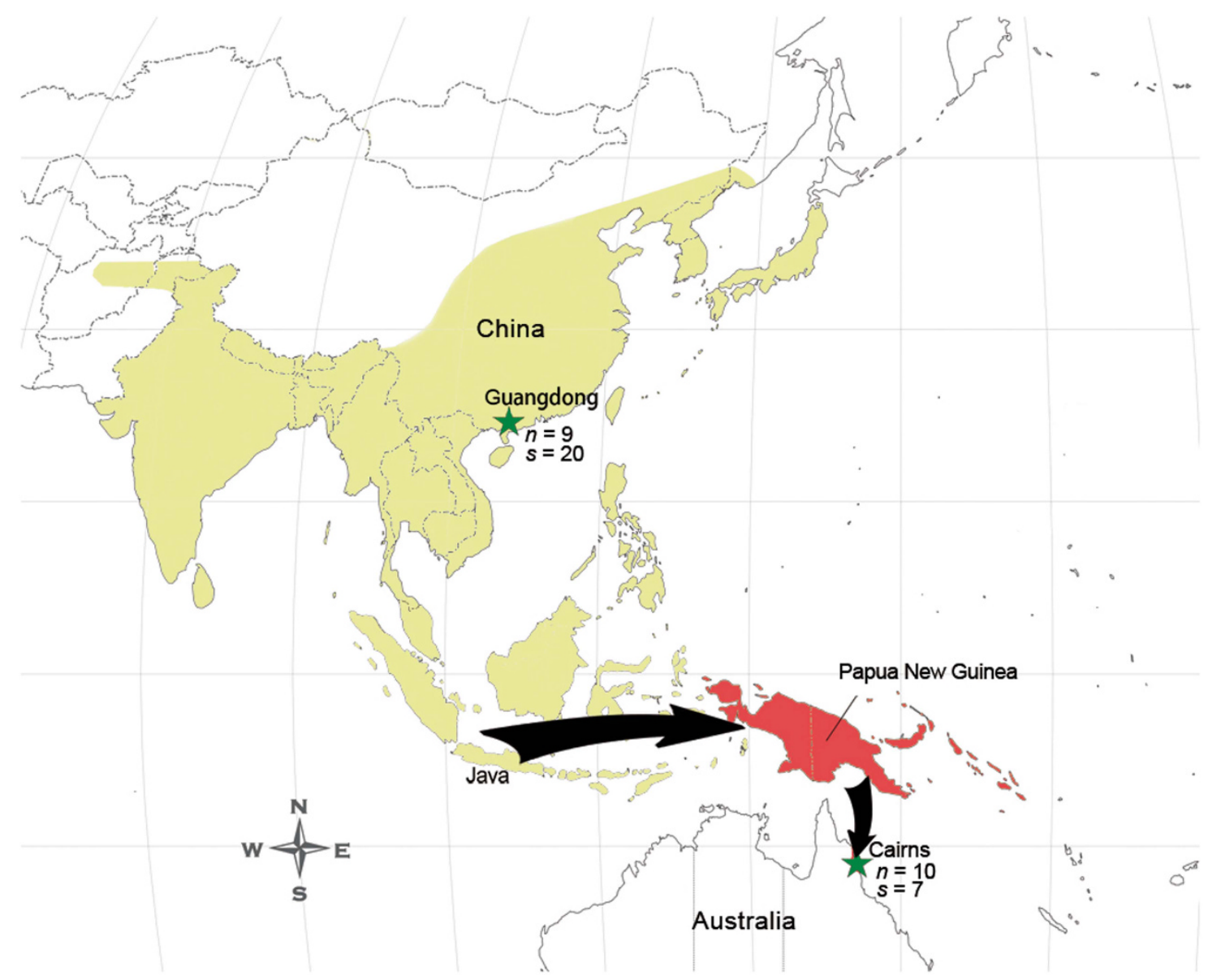

Figure 1 The distribution of Apis cerana showing its natural range (yellow), its invasive range (red) and the suspected route (black arrows) of Australia's invasive population, first introduced from Java, Indonesia, to Papua New Guinea and from there introduced accidently to Australia in 2007. The two populations analysed in this study are indicated, together with the number of sex alleles present $(s)$ and number of colonies $(n)$ for estimates of mating frequency.

3, fertilized eggs producing males instead of workers), to represent high and very high likelihoods respectively, that a colony would have reduced reproductive output and survival. Exactly how honey bee fitness declines with decreasing brood viability is poorly understood. Colonies with low levels of DMP probably suffer only marginal loss of fitness (Cook and Crozier, 1995), but experimental manipulations in Apis mellifera indicate that worker population or colony survival declines significantly at $\geqslant 25 \%$ DMP (Woyke, 1980; Tarpy and Page, 2002).

\section{RESULTS}

\section{Mating frequency of $A$. cerana queens}

In both native and invasive populations mating frequency was high (China: $k=29.0 \pm 7.78$ (s.d.), $m=24.6 \pm 8.83, n=9$, and Australia: $k=27.4 \pm 7.81, m=21.0 \pm 8.29, n=10)$ and nondetection rates were low (China: $d_{p}=0.0006$, Australia: 0.0013; Table 1). Given the observed mating frequency in our invasive population, and assuming a source population with 20 alleles at equilibrium frequency and random mating, we estimated that a founding $A$. cerana queen would arrive in a new location carrying 15 (range 9-20, s.d. = 1.4) unique sex alleles in stored sperm, or $75 \%$ of that present in the original population.

We found no significant difference in the observed queen mating frequency between our native and invasive populations $(t=0.447$, d.f. $=17, P=0.66)$. Given the high within-population variance in mating frequency (China: range 19-46, Australia: range 16-42), our power to detect a true effect size as small as that observed was low (post hoc analysis: Cohen's $d=0.205$, power $=0.06$ ), but it is clear that mating frequencies are similar in the native Chinese and invasive Australian populations and any difference is unlikely to be of biological importance.

\section{Variance in brood viability in invasive populations}

The mean incidence of DMP in a population is a function of sex allele number $(s)$ and relative frequency, independent of queen mating frequency (Figure 2). Where invasive populations have reduced $s$, or heavily skewed sex allele frequencies, they endure a higher mean incidence of DMP than native-range populations (Figure 2).

Increased queen mating frequency $(k)$ always reduces the variance around the mean DMP, although the magnitude of this change and the extent to which it affects the proportion of colonies with very high DMP varies depending on sex allele number and relative frequencies (Figure 2). Figure 3 illustrates the relationship between mating frequency and incidences of high DMP for simulated native-range and invasive populations of $A$. cerana. In native-range populations (ie, many sex alleles in equal frequency), the risk of very high DMP is always low, and no colonies exceed 35\% DMP under either moderate $(k=6)$ or extreme $(k=27)$ polyandry (Figures $3 \mathrm{~b}$ and $\mathrm{c})$. In contrast, in a newly founded invasive population with a small number of alleles at unequal frequencies, moderate polyandry markedly decreases the proportion of colonies experiencing very high DMP (10.4\% of colonies have $\geqslant 35 \%$ DMP), relative to monandry ( $46.7 \%$ of colonies), 
Table 1 Estimates of Apis cerana queen mating frequency

\begin{tabular}{|c|c|c|c|c|c|c|}
\hline Location & Colony ID & Sample size (n) & Observed mating frequency $(\mathrm{k})$ & Effective mating frequency $(\mathrm{m})$ & $95 \% \mathrm{Cl}$ around $\mathrm{m}$ & Nondetection error $\left(\mathrm{d}_{\mathrm{p}}\right)$ \\
\hline & A2 & 96 & 31 & 27.98 & 2.92 & \\
\hline & A4 & 96 & 28 & 19.76 & 1.65 & \\
\hline & A5 & 96 & 30 & 16.06 & 1.18 & \\
\hline & A6 & 96 & 42 & 38.33 & 4.89 & \\
\hline & A9 & 96 & 18 & 16.17 & 1.19 & \\
\hline & A10 & 96 & 16 & 7.78 & 0.36 & \\
\hline Mean \pm s.d. & & $96.0 \pm 0.00$ & $27.4 \pm 7.81$ & $21.0 \pm 8.29$ & $1.96 \pm 1.26$ & \\
\hline \multirow[t]{5}{*}{ China } & $\mathrm{C} 1$ & 84 & 24 & 17.98 & 1.64 & 0.0006 \\
\hline & $\mathrm{C} 2$ & 84 & 27 & 23.56 & 2.55 & \\
\hline & $\mathrm{C7}$ & 86 & 32 & 32.35 & 4.29 & \\
\hline & $\mathrm{c8}$ & 86 & 45 & 41.55 & 6.46 & \\
\hline & C9 & 86 & 24 & 18.65 & 1.74 & \\
\hline Mean \pm s.d. & & $85.3 \pm 1.00$ & $29.0 \pm 7.78$ & $24.6 \pm 8.83$ & $2.9 \pm 1.70$ & \\
\hline
\end{tabular}

Included for each population is the average ( \pm s.d.) for: worker pupae sample size per colony $(n)$, observed mating frequency $(k)$, effective mating frequency $(m)$, $95 \%$ confidence intervals $(C l s)$ of $m$ and the nondetection error at population level $\left(d_{p}\right)$.

whereas extreme polyandry lowers the incidence further still $(4.2 \%$, Figures $3 \mathrm{~d}-\mathrm{f}$ ). Similarly, once an invasive population is established and sex alleles have stabilized at equal frequency, extreme polyandry leads to lower incidences of high DMP $(1.1 \%$ of colonies at $\geqslant 25 \%$ DMP and none at $\geqslant 35 \%$ DMP) than either moderate polyandry $(22.8 \%$ and $0.7 \%$ at $\geqslant 25 \%$ DMP and $\geqslant 35 \%$ DMP, respectively) or monandry $(28.9 \%$ colonies at $\geqslant 35 \%$ DMP; Figures $3 g-i)$. Clearly, however, the role of extreme polyandry in reducing the incidence of costly levels of DMP depends on both the mean DMP and the DMP level at which fitness is affected. This is because the effect of increasing mate number is to reduce variance in colonies' DMP frequency around the mean (Figures 2 and 3). If the mean DMP is at or above a critical value determining colony fitness (as in our simulations of Australia's invasive A. cerana), then extreme polyandry succeeds in reducing the incidence of high DMP. If mean DMP is higher than this critical value then high levels of $k$ provide little relief. In such cases, colony survivorship may be so low that an incipient population is doomed regardless of mating frequency.

\section{DISCUSSION}

We propose that the extreme polyandry of $A$. cerana contributes to their ability to establish invasive populations in at least two ways. First, multiple-mated queens carry the stored sperm of, on average, 27 males that is sufficient to carry $75 \%$ of the genetic diversity at the sex locus present in their parent population. These alleles must find their way into new queens or males if they are to persist in the population, and many will presumably be lost in the early generations following a founder event (Leberg, 1992; Greenbaum et al., 2014; Gloag et al., 2016). Nevertheless, under high levels of polyandry, the probability that one or few founders will succeed in transferring enough allelic diversity at the sex locus to establish a thriving population far exceeds that of species whose queens mate just once or a few times.
Second, extreme polyandry reduces the variance in brood viability experienced by colonies in invasive populations following founder events. The role of polyandry in reducing such variance has been described previously (Page, 1980; Page and Marks, 1982; Palmer and Oldroyd, 2000), but the relative advantage of extreme mating frequencies, over moderate mating frequencies, in reducing DMP variance is marginal in native-range populations where allele diversity is high and allele frequencies are equal (Figures $2 \mathrm{a}$ and $3 \mathrm{a}-\mathrm{c}$ ). This has led to the view that mating more than around six times provides little fitness benefit in terms of brood viability (Palmer and Oldroyd, 2000; Figure 2a). However, new populations founded by few individuals, or populations that have otherwise experienced a dramatic bottleneck, are likely to have both relatively low allele number and unequal allele frequencies (Leberg, 1992; Greenbaum et al., 2014; Gloag et al., 2016). Under these conditions, extreme polyandry can reduce DMP variance among colonies, relative to lower mate numbers, to an extent that seems likely to have biological significance. For example, the proportion of colonies enduring high DMP (25-50\% DMP) drops by $20 \%$ under the allele richness and frequency conditions experienced by Australia's invasive A. cerana population (both after founding and when established, Figures $3 \mathrm{~d}-\mathrm{i}$ ). Some benefit of extreme polyandry following founder events will hold for all but the most extreme cases of allele loss or skew (eg, where mean DMP is so high as to negate the benefit of reduced variance from the mean).

Small populations are vulnerable to extinction from stochastic events, so the more colonies that survive to reproduce the more likely an invasive population will persist (Blackburn et al., 2015). In social hymenopteran species, DMP slows colony growth and imposes a fitness cost, both via the wasted resources of laying and partially rearing inviable or sterile males and the increased risk that colonies never attain a workforce large enough to produce daughter colonies (Tarpy and Page, 2001, 2002; Dlugosch and Parker, 2008). It has been shown that DMP can reduce colony growth in bumble bees 

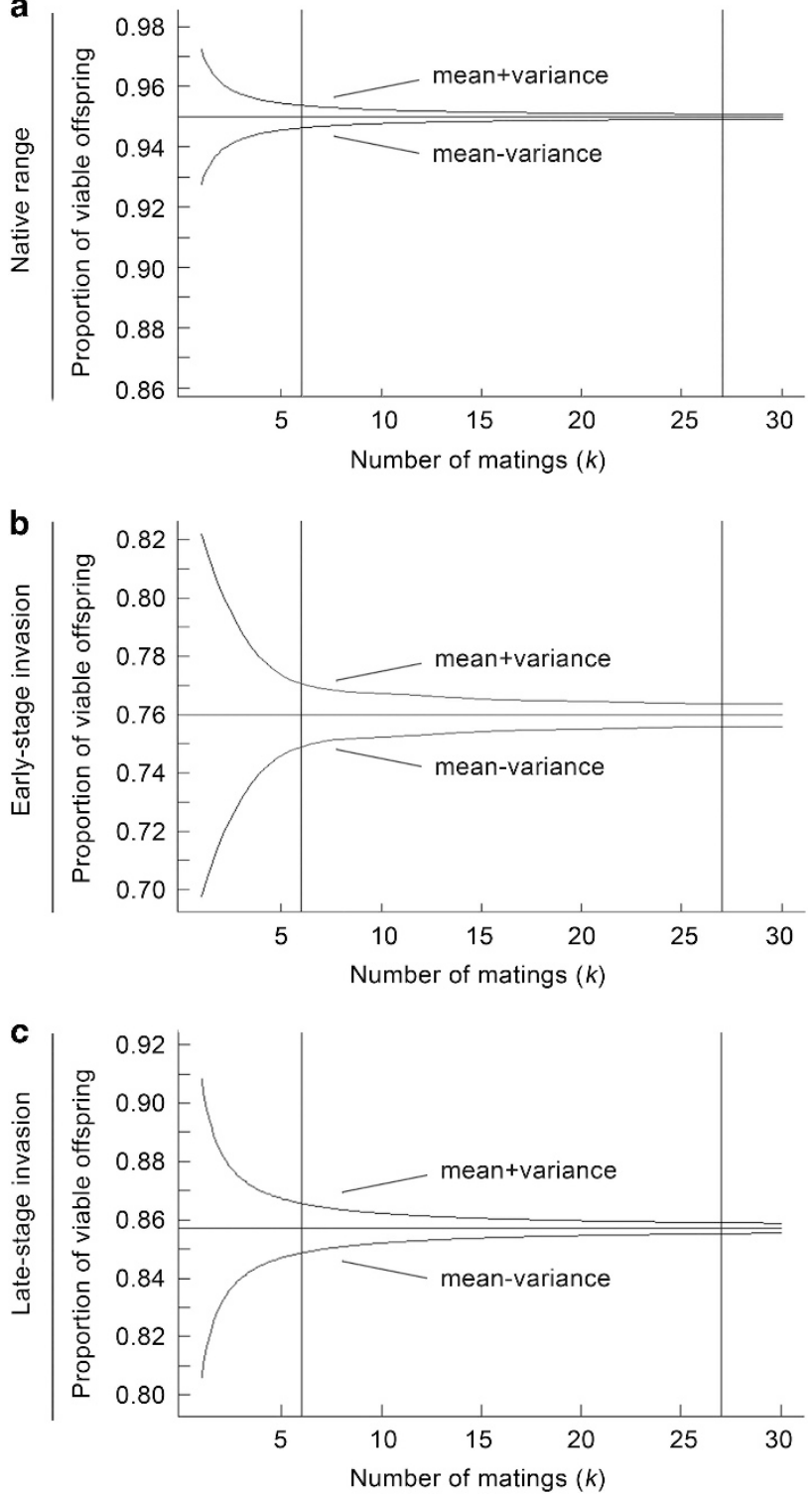

Figure 2 The relationship between mean and variance in brood viability and mating frequency under different scenarios of sex allele number and frequencies in a population. (a) Native-range population with 20 sex alleles at equal frequency. (b) Early-stage invasive population with 7 sex alleles at unequal frequency. (c) Late-stage invasive population with 7 sex alleles at equal frequency. Vertical lines indicate an illustrative 'moderate' mating frequency $(k=6)$ and 'extreme' mating frequency $(k=27)$.

(Whitehorn et al., 2009) and increase mortality during colony founding in ants (Ross and Fletcher, 1986). Tarpy and Page (2002) found that increasing levels of DMP in A. mellifera are correlated with decreases in several proxies of colony fitness, including worker population size, brood area and area of stored food, as well as dramatically increasing the likelihood of colony mortality over winter. How these effects translate to tropical honey bees such as invasive $A$. cerana is uncertain, but it seems safe to assume that very high DMP compromises the rate at which colonies produce daughter swarms, thereby slowing growth of the invasive population and increasing its vulnerability to stochastic processes. In worst case scenarios (say DMP of above 25 or $35 \%$ ) colonies may be incapable of reproducing at all (Cook and Crozier 1995). Notably, in our previous simulations of changes in sex allele frequency in Australia's invasive A. cerana population (Gloag et al., 2016), only models that assumed significant fitness costs of DMP produced good fits to empirical data. Thus, it is likely that high DMP incurs fitness costs to A. cerana colonies, and that the reduced variance in DMP afforded by extreme polyandry has aided A. cerana's invasive spread to Australia and other Pacific regions.

Given that the benefits of polyandry are most pronounced in bottlenecked populations, we might imagine that polyandrous social insects such as honey bees adopt a flexible mating strategy in which queen's mate number increases as the risks of inbreeding and DMP increase. Such flexibility in mating behaviour in response to inbreeding risk has been documented in some vertebrates (Arct et al., 2015; Nichols et al., 2015). In polyandrous social insect species, there are often significant differences in mating frequency among populations (A. mellifera: Franck et al., 2000; Kraus et al., 2004; El-Niweiri and Moritz, 2011; A. cerana: DeFelice et al., 2015 and the ant Lasius niger: Corley and Fjerdingstad, 2011). This phenomenon has been postulated as an adaptive response to reduce inbreeding (Ratnieks, 1990; Tregenza and Wedell, 2002; Michalczyk et al., 2011). Nonetheless, there are several instances where small populations of social insects show no evidence of increased mating frequency (eg, an isolated population of feral A. mellifera in New York state, Tarpy et al., 2015, and invasive Vespula pensylvanica in Hawaii, Hanna et al., 2014). We did not detect interpopulation variation in mating frequency between the invasive Australian A. cerana (where inbreeding risk is high) and a native Chinese population (where inbreeding risk is low), consistent with the view that $A$. cerana mating frequency is not a labile trait that can rapidly evolve or adapt to current circumstances (Tarpy et al., 2015). Notably, however, in both populations, variation among individual queens was high, with some queens having more than twice the mating frequency as others in the same population. Whether mate number is ever constrained in A. cerana due to ecological conditions remains to be determined. For example, interspecific mating has been detected between A. cerana and A. mellifera in both China and Australia (Remnant et al., 2014), and incurs a high cost to both species (Gloag et al., 2017). Such reproductive interference might constrain attempts by queens to increase mate number.

The consequences of extreme multiple mating on A. cerana's invasive potential provide a clear example of how polyandry may increase population viability and mitigate extinction risk, and hence illustrates well the general principal that mating systems may influence the fate of populations (Holman and Kokko, 2013). In this study, we have focused on the consequences of extreme polyandry for sex locusdetermined brood viability in newly founded populations. The extent to which polyandry contributes to invasiveness via sex locus effects in other invasive Hymenoptera remains to be investigated, but at least some other successful invaders that have founded populations from single queens are also polyandrous, including Vespa velutina in Europe and Korea (Arca et al., 2015) and Acromyrmex octospinosus in Guadeloupe (Mikheyev, 2008). Increased brood viability may not, however, be the only route via which polyandry effects the survival of invasive populations. Notably, genome-wide loss of genetic diversity in populations founded by just one or few colonies will also be less severe when queens are polyandrous than when they are singly mated (Arca et al., 2015; Darrouzet et al., 2015). Given that genetic diversity within honey bee colonies increases both disease resistance and task partitioning (Oldroyd and Fewell, 2008), polyandry may also have served A. cerana and other invasive Apis in this respect (eg, Africanized A. mellifera in the Americas, Tarpy et al., 2010, and Apis florea in East Africa and the Arabian Peninsula, Moritz et al., 2009). However, high diversity at loci other than the sex loci is not necessarily beneficial to 

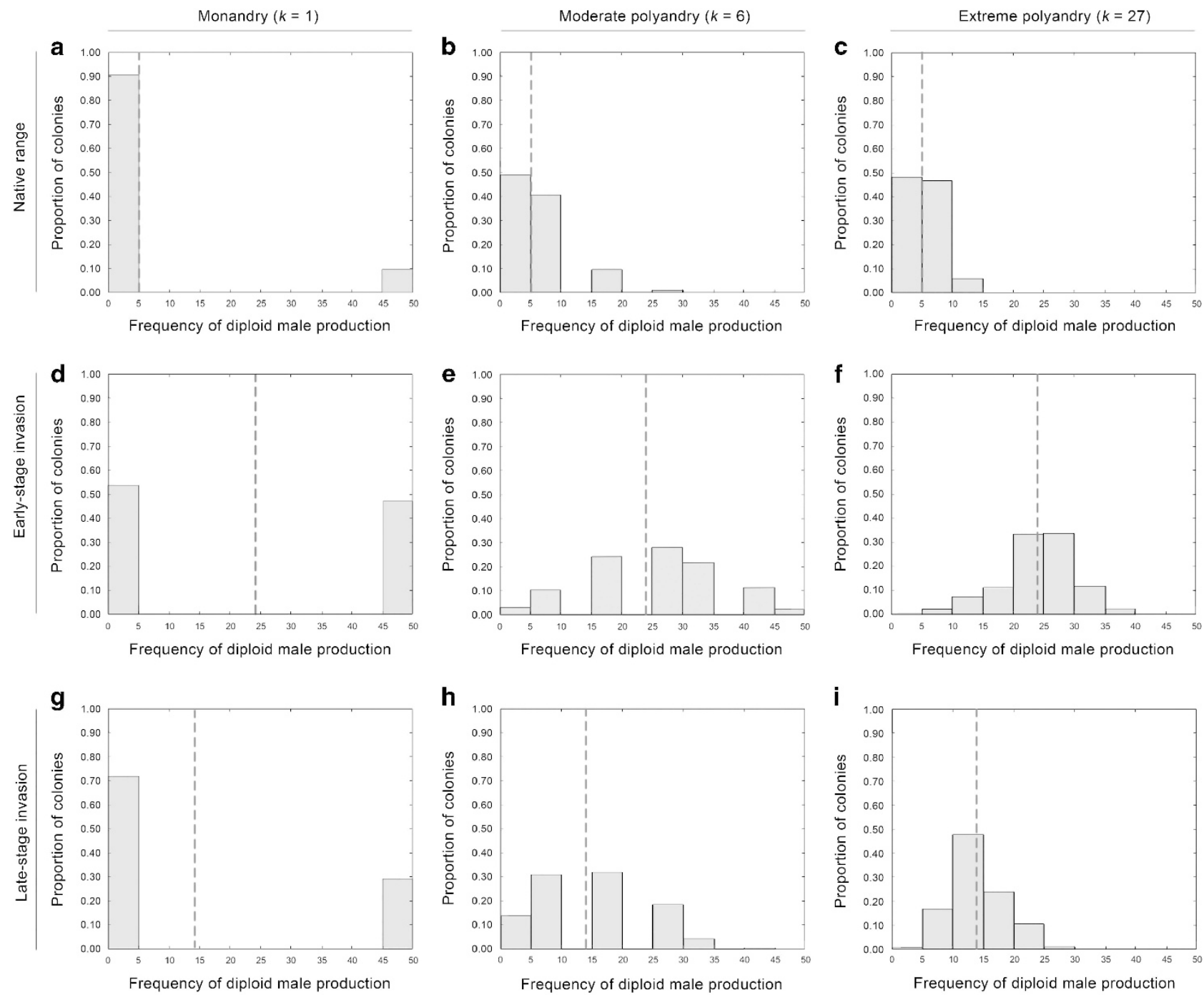

Figure 3 Effect of queen mating frequency on the incidence of diploid male production (DMP) for different scenarios of sex allele number and frequencies in a population. Dotted lines indicate the average proportion of DMP in the population that is independent of mating frequency. (a) 'Monandry' ( $k=1$ ), (b) 'moderate polyandry' $(k=6)$ and (c) 'extreme polyandry' $(k=27)$ when $s=20$ at equal frequency, as typical of a native-range population; (d) 'monandry' $(k=1)$, (e) 'moderate polyandry' $(k=6)$ and (f) 'extreme polyandry' $(k=27)$ when $s=7$ at unequal frequency, as occurs in the early stages of an invasive population after a founder event; (g) 'monandry' $(k=1)$, (h) 'moderate polyandry' $(k=6)$ and (i) 'extreme polyandry' $(k=27)$ when $s=7$ at equal frequency, as occurs in the late stages of an invasive population after a founder event.

invasiveness; for example, low intercolony aggression and supercolony formation in invasive populations of the Argentine ant (Linepithema humile) have been associated with a loss of either overall genetic diversity (Tsutsui et al., 2000) or diversity at relevant 'recognition loci' (Giraud et al., 2002). Queen polyandry may also have other significant, nongenetic, consequences for small invasive populations of social insects (eg, effects on queen survival during mating, or on effective population size) that remain to be investigated.

The primary selective forces driving the evolution of polyandry in some social insect lineages likely pertain to the benefits of withincolony genetic diversity on colony fitness (Oldroyd and Fewell, 2008). Our study supports the view that, once evolved, extreme polyandry can also have important ecological consequences for species by increasing their ability to found new populations from few individuals. In the case of A. cerana, extreme polyandry is one of a suite of traits that has facilitated the species conquest of a new continent, Australia, from the accidental introduction of just one or a few colonies.

\section{DATA ARCHIVING}

Genotype data for workers from Australia and China have been submitted to Dryad: doi:10.5061/dryad.2bk07.

\section{CONFLICT OF INTEREST}

The authors declare no conflict of interest.

\section{ACKNOWLEDGEMENTS}

GD was supported by China Scholarship Council (No.201503250033), the Agricultural Science and Technology Innovation Program (CAAS-ASTIP-2015IAR) and the Central Public-interest Scientific Institution Basal Research Found (No. 16078). RSG was supported by the University of Sydney Postdoctoral Fellowship. Research funding came from Australian Research Council DP150101985 to BPO and RSG. We thank the Queensland Department of Agriculture and Fisheries, Australian government Department of Agriculture and Water Resources and Roy Swenson for their assistance in collecting samples. 
Arca M, Mougel F, Guillemaud T, Dupas S, Rome Q Perrard A et al (2015). Reconstructing the invasion and the demographic history of the yellow-legged hornet, Vespa velutina, in Europe. Biol Invasions 8: 2357-2371.

Arct A, Drobniak SM, Cichoń M (2015). Genetic similarity between mates predicts extrapair paternity-a meta-analysis of bird studies. Behav Ecol 26: 959-968.

Baer B, Schmid-Hempel P (1999). Experimental variation in polyandry affects parasite loads and fitness in a bumble-bee. Nature 397: 161-164.

Barth MB, Moritz RFA, Kraus FB (2014). The evolution of extreme polyandry in social insects: insights from army ants. PLoS One 9: e105621.

Berggren A (2001). Colonization success in Roesel's bush-cricket Metrioptera roeseli: the effects of propagule size. Ecology 82: 274-280.

Beye M, Hasselmann M, Fondrk MK, Page RE, Omholt SW (2003). The gene csd is the primary signal for sexual development in the honeybee and encodes an SR-type protein. Cell 114: 419-429.

Blackburn TM, Lockwood JL, Cassey P (2015). The influence of numbers on invasion success. Mol Ecol 24: 1942-1953.

Boomsma JJ (2009). Lifetime monogamy and the evolution of eusociality. Phil Trans $R$ Soc B 364: 3191-3207.

Boomsma JJ, Ratnieks FL (1996). Paternity in eusocial Hymenoptera. Phil Trans $R$ Soc $B$ 351: 947-975.

Cook JM, Crozier RH (1995). Sex determination and population biology in the Hymenoptera. Trends Ecol Evol 10: 281-286.

Corley M, Fjerdingstad EJ (2011). Mating strategies of queens in Lasius niger ants-is environment type important? Behav Ecol Sociobiol 65: 889-897.

Cornwallis CK, West SA, Davis KE, Griffin AS (2010). Promiscuity and the evolutionary transition to complex societies. Nature 466: 969-972.

Darrouzet E, Gevar J, Guignard Q, Aron S (2015). Production of early diploid males by European colonies of the invasive hornet Vespa velutina nigrithorax. PLoS One 10: e0136680.

DeFelice D, Ross C, Simone-Finstrom M, Warrit N, Smith D, Burgett M et al. (2015). Geographic variation in polyandry of the eastern honey bee, Apis cerana, in Thailand. Insect Soc 62: 37-42.

Dlugosch KM, Parker IM (2008). Founding events in species invasions: genetic variation, adaptive evolution, and the role of multiple introductions. Mol Ecol 17: 431-449.

El-Niweiri MAA, Moritz RFA (2011). Mating in the rain? Climatic variance for polyandry in the honeybee (Apis mellifera jemenitica). Popul Ecol 53: 421-427.

Fjerdingstad EJ, Boomsma JJ (2000). Queen mating frequency and relatedness in young Atta sexdens colonies. Insect Soc 47: 354-356.

Franck P, Koeniger N, Lahner G, Crewe R, Solignac M (2000). Evolution of extreme polyandry: an estimate of mating frequency in two African honeybee subspecies, Apis mellifera monticola and A.m.scutellata. Insect Soc 47: 364-370.

Giraud T, Pedersen JS, Keller L (2002). Evolution of supercolonies: the Argentine ants of southern Europe. Proc Natl Acad Sci USA 9: 6075-6079.

Gloag R, Ding G, Christie JR, Buchmann G, Beekman M, Oldroyd BP (2016). An invasive social insect overcomes genetic load at the sex locus. Nat Ecol Evol 1: 0011.

Gloag R, Tan K, Wang Y, Song W, Luo W, Buchman G et al. (2017). No evidence of queen thelytoky following interspecific crosses of the honey bees Apis cerana and Apis mellifera. Insect Soc 64: 241.

Goodisman MAD, Matthews RW, Crozier RH (2002). Mating and reproduction in the wasp Vespula germanica. Behav Ecol Sociobiol 51: 497-502.

Greenbaum G, Templeton AR, Zarmi Y, Bar-David S (2014). Allelic richness following population founding events-a stochastic modeling framework incorporating gene flow and genetic drift. PLOS ONE 9: e115203.

Hanna C, Cook ED, Thompson AR, Dare LE, Palaski AL, Foote D et al. (2014). Colony social structure in native and invasive populations of the social wasp Vespula pensylvanica. Biol Invasions 16: 283-294.

Hasselmann M, Beye M (2004). Signatures of selection among sex-determining alleles of the honey bee. Proc Natl Acad Sci USA 101: 4888-4893.

Hasselmann M, Vekemans X, Pflugfelder J, Koeniger N, Koeniger G, Tingek S et al. (2008). Evidence for convergent nucleotide evolution and high allelic turnover rates at the complementary sex determiner gene of Western and Asian honeybees. Mol Biol Evol 25 696-708.

Heimpel GE, de Boer JG (2008). Sex determination in the Hymenoptera. Annu Rev Entomol 53: 209-230.

Holman L, Kokko H (2013). The consequences of polyandry for population viability, extinction risk and conservation. Phil Trans R Soc B 368: 20120053.

Hughes WOH, Oldroyd BP, Beekman M, Ratnieks FLW (2008). Ancestral monogamy shows kin selection is the key to the evolution of eusociality. Science 320: 1213-1216.

Jones JC, Myerscough MR, Graham S, Oldroyd BP (2004). Honey bee nest thermoregulation: diversity promotes stability. Science 305: 402-404.

Jones OR, Wang J (2010). COLONY: a program for parentage and sibship inference from multilocus genotype data. Mol Ecol Resour 10: 551-555.

Koetz AH (2013). Ecology, behaviour and control of Apis cerana with a focus on relevance to the Australian incursion. Insects 4: 558-592.

Kraus FB, Neumann P, van Praagh J, Moritz RFA (2004). Sperm limitation and the evolution of polyandry in the honeybee (Apis mellifera L.). Behav Ecol Sociobiol $\mathbf{5 5}$ 494-501.
Kronauer DJ, Johnson RA, Boomsma JJ (2007). The evolution of multiple mating in army ants. Evolution 61: 413-422.

Leberg PL (1992). Effects of population bottlenecks on genetic diversity as measured by allozyme electrophoresis. Evolution 46: 477-494.

Mattila HR, Reeve HK, Smith ML (2012). Promiscuous honey bee queens increase colony productivity by suppressing worker selfishness. Curr Biol 22: 2027-2031.

Mattila HR, Seeley TD (2007). Genetic diversity in honey bee colonies enhances productivity and fitness. Science 317: 362-364.

Michalczyk Ł, Millard AL, Martin OY, Lumley AJ, Emerson BC, Chapman T et al. (2011). Inbreeding promotes female promiscuity. Science 333: 1739-1742.

Mikheyev AS (2008). History, genetics and pathology of a leafcutting ant introduction: a case study of the Guadeloupe invasion. Biol Invasions 10: 467-473.

Moritz RFA, Haddad N, Bataieneh A, Shalmon B, Hefetz A (2009). Invasion of the dwarf honeybee Apis florea into the near East. Biol Invasions 12: 1093-1099.

Nichols HJ, Cant MA, Sanderson JL (2015). Adjustment of costly extra-group paternity according to inbreeding risk in a cooperative mammal. Behav Ecol 26 1486-1494.

Nielsen R, Tarpy DR, Reeve HK (2003). Estimating effective paternity number in social insects and the effective number of alleles in a population. Mol Ecol 12: 3157-3164.

Oldroyd BP, Clifton MJ, Parker K, Wongsiri S, Rinderer TE, Crozier RH (1998). Evolution of mating behavior in the genus Apis and an estimate of mating frequency in Apis cerana (Hymenoptera: Apidae). Ann Entomol Soc Am 91: 700-709.

Oldroyd BP, Fewell JH (2008). Large fitness benefits from polyandry in the honey bee, Apis mellifera. Trends Ecol Evol 23: 59-60.

Palmer K, Oldroyd BP (2000). Evolution of multiple mating in the genus Apis. Apidologie 31: 235-248.

Page R (1980). The evolution of multiple mating behaviour by honey bee queens (Apis mellifera L.). Genetics 96: 263-273.

Page R, Marks RW (1982). The population genetics of sex determination in honey bees: random mating in closed populations. Heredity 48: 263-270.

Ratnieks FW (1988). Reproductive harmony via mutual policing by workers in eusocial Hymenoptera. Am Nat 132: 217-236.

Ratnieks FW (1990). The evolution of polyandry by queens in social hymenoptera: the significance of the timing of removal of diploid males. Behav Ecol Sociobiol 28 343-348.

Remnant EJ, Koetz A, Tan K, Hinson E, Beekman M, Oldroyd BP (2014). Reproductive interference between honey bee species in artificial sympatry. Mol Ecol 23: 1096-1107.

Ross KG, Fletcher DJC (1986). Diploid male production-a significant colony mortality factor in the fire ant Solenopsis invicta (Hymenoptera: Formicidae). Behav Ecol Sociobiol 4: 283-291.

Seeley TD, Tarpy DR (2007). Queen promiscuity lowers disease within honeybee colonies. Proc $R$ Soc B Biol Sci 274: 67-72.

Solignac M, Vautrin D, Loiseau A, Mougel F, Baudry E, Estoup A et al. (2003). Five hundred and fifty microsatellite markers for the study of the honeybee (Apis mellifera L.) genome. Mol Ecol Notes 3: 307-311.

Takahashi J, Shimizu S, Koyama S, Kimura K, Shimuzu I, Yoshida T (2009). Variable microsatellite loci isolated from the Asian honeybee, Apis cerana (Hymenoptera; Apidae). Mol Ecol Resour 9: 819-821.

Tarpy DR, Caren JR, Delaney DA, Sammataro D, Finley J, Loper GM et al. (2010). Mating frequencies of Africanized honey bees in the south western USA. J Apicul Res 49: 302-310

Tarpy DR, Delaney DA, Seeley TD (2015). Mating frequencies of honey bee queens (Apis mellifera L.) in a population of feral colonies in the Northeastern United States. PLoS ONE 10: e0118734

Tarpy DR, Nielsen R, Nielsen DI (2004). A scientific note on the revised estimates of effective paternity frequency in Apis. Insect Soc 51: 203-204.

Tarpy DR, Page RE Jr (2001). The curious promiscuity of queen honey bees (Apis mellifera): evolutionary and behavioral mechanisms. Ann Zool Fennici 38 $255-265$

Tarpy DR, Page RE Jr (2002). Sex determination and the evolution of polyandry in honey bees (Apis mellifera). Behav Ecol Sociobiol 52: 143-150.

Tregenza T, Wedell N (2002). Polyandrous females avoid costs of inbreeding. Nature 415 71-73.

Tsutsui ND, Suarez AV, Holway DA, Case TJ (2000). Reduced genetic variation and the success of an invasive species. Proc Natl Acad Sci USA 11: 5948-5953.

van Wilgenburg E, Driessen G, Beukeboom LW (2006). Single locus complementary sex determination in Hymenoptera: an 'unintelligent' design? Front Zool 3: 1-15.

Walsh PS, Metzger DA, Higuchi R (1991). Chelex-100 as amedium for simple extraction of DNA for PCR-based typing from forensic material. Biotechniques 10: 506-513.

Whitehorn PR, Tinsley MC, Brown MJF, Darvill B, Goulson D (2009). Impact of inbreeding on bumblebee colony fitness under field conditions. BMC Evol Biol 9: 152 .

Woyke J (1980). Effect of sex allele homo-heterozygosity on honeybee colony populations and on their honey production. I. Favourable development conditions and unrestricted queens. J Apic Res 19: 51-63.

Zayed A, Packer L (2005). Complementary sex determination substantially increases extinction proneness of haplodiploid populations. Proc Natl Acad Sci USA 102 10742-10746.

Supplementary Information accompanies this paper on Heredity website (http://www.nature.com/hdy) 